\title{
Online Scheduling with Interval Conflicts
}

\author{
Magnús M. Halldórsson*1, Boaz Patt-Shamir ${ }^{\dagger 2}$, and Dror Rawitz ${ }^{2}$ \\ 1 School of Computer Science, Reykjavik University, Menntavegur 1, 101 \\ Reykjavik, Iceland. \\ mmh@ru.is \\ 2 School of Electrical Engineering, Tel Aviv University, Tel Aviv 69978, Israel. \\ \{boaz, rawitz\}@eng.tau.ac.il
}

\begin{abstract}
In the problem of Scheduling with Interval Conflicts, there is a ground set of items indexed by integers, and the input is a collection of conflicts, each containing all the items whose index lies within some interval on the real line. Conflicts arrive in an online fashion. A scheduling algorithm must select, from each conflict, at most one survivor item, and the goal is to maximize the number (or weight) of items that survive all the conflicts they are involved in. We present a centralized deterministic online algorithm whose competitive ratio is $O(\lg \sigma)$, where $\sigma$ is the size of the largest conflict. For the distributed setting, we present another deterministic algorithm whose competitive ratio is $2 \lg \sigma$, in the special contiguous case, in which the item indices constitute a contiguous interval of integers. Our upper bounds are complemented by two lower bounds: one that shows that even in the contiguous case, all deterministic algorithms (centralized or distributed) have competitive ratio $\Omega(\lg \sigma)$, and that in the non-contiguous case, no deterministic oblivious algorithm (i.e., a distributed algorithm that does not use communication) can have a bounded competitive ratio.
\end{abstract}

1998 ACM Subject Classification F.2.2 Analysis of Algorithms and Problem Complexity: Nonnumerical Algorithms and Problems - Computations on discrete structures and Sequencing and scheduling; G.2.1 Discrete Mathematics: Combinatorics - Combinatorial algorithms

Keywords and phrases online scheduling, online set packing, interval conflicts, competitive analysis, compound tasks, distributed algorithms

Digital Object Identifier 10.4230/LIPIcs.STACS.2011.472

\section{Introduction}

We study the following abstract problem, which we call Scheduling with Interval Conflicts. There is a universe $U$ of $n$ items, each with an integer identifier. The input is a collection $\mathcal{C}$ of conflicts, where each conflict $C \in \mathcal{C}$ is a set containing all the items of $U$ within some interval on the real line. A conflict represents an event where the specified items compete for a resource that can be granted to only one item. Conflict resolution is carried out by a scheduling algorithm that decides which item survives: all other items in the conflict set are eliminated. The goal of the scheduling algorithm is to maximize the number (or weight) of items that survive all their conflicts.

Scheduling with Interval Conflicts arises naturally in some scenarios. One interpretation of the model is when we have a set of permanently-running stations that may interfere

* Supported in part by the Iceland Research Fund (grant 90032021).

$\dagger$ Supported in part by the Israel Science Foundation (grant 1372/09) and by a grant from Israel Ministry of Science and Technology.

(c) (1) () $($ ) Magnús M. Halldórsson, Boaz Patt-Shamir, and Dror Rawitz; cc. 28th Symposium on Theoretical Aspects of Computer Science (STACS'11). Editors: Thomas Schwentick, Christoph Dürr; pp. 472-483 Leibniz International Proceedings in Informatics

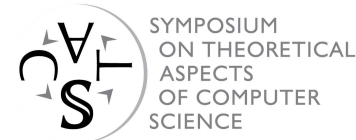
LI I ICS Schloss Dagstuhl - Leibniz-Zentrum für Informatik, Dagstuhl Publishing, Germany 
only with other neighboring stations, where the underlying metric space is a line, and the interference range in each direction may change in every step. In each step we need to choose a station that will win the current conflict, if any. The goal is to maximize the number of stations that never fail.

Another example for our model are tasks that must be processed by a few boundedcapacity servers located at different sites on the Internet. The tasks are sent to these servers in the same order, and due to varying congestion conditions in the network, they arrive at the servers with varying burstiness: for example, the input to server $A$ may be such that at step $t$ task $i$ arrives, at step $t+1$ tasks $i+1, i+2, i+3$ arrive together, at step $t+2$ no task arrives etc. The input to another server $B$ may exhibit a different burst structure, e.g., tasks $i$ and $i+1$ arrive together, and tasks $i+2$ and $i+3$ arrive together. Assume that the servers can process only one task at a step, and tasks cannot be stored for later processing. Then a time step in which more than a single task arrives can be represented as an interval conflict. The main question in our model is which tasks to process and which to drop, so as to maximize the total number of tasks that receive all the processing they require.

Finally, consider multiple streams of data-frames (e.g., video frames) that need to be transmitted across the Internet. Since data frames are typically too large to fit in a single packet, the frames are broken into a number of packets, and reconstructed at the receiver. However, if a packet is lost in transit, its whole constituent frame (i.e., item) becomes useless. Interval conflicts arise if the streams pass through a congested router which can forward only one packet from each burst of packets that arrive together (all other packets are dropped).

\section{Problem variants}

In some cases, conflicts need to be resolved without knowledge of other conflicts (for example, if conflicts arrive in different locations, or if the conflict resolution protocol must be stateless). We call this variant oblivious (or distributed) scheduling. In some other cases, all previous conflicts and their outcomes are known to the algorithm when a new conflict arrives. We call this variant sequential (or centralized) scheduling. Note that both oblivious and sequential scheduling are online, i.e., no information about future conflicts is available to the algorithm (the offline variant of the problem is when all conflicts are given ahead of time).

An interesting special case of interval conflicts is when the universe of items contains no gaps, i.e., the items have identifiers $i_{0}, i_{0}+1, \ldots, i_{0}+n-1$ (in general, item identifiers are only required to be totally ordered). We refer to this as the contiguous case.

\subsection{Our Contribution}

In this paper we introduce and formalize the problem of Scheduling with Interval Conflict (abbreviated SIC below), and give deterministic online algorithms and lower bounds on the competitive ratio of deterministic algorithms. We start off with the special case of contiguous conflicts. It turns out that contiguous conflicts allow for an oblivious (and hence distributed) algorithm, guaranteeing competitive ratio of $O(\lg \sigma)$, where $\sigma$ is the maximal number of items in a conflict. However, no competitive oblivious algorithm exists if item identifiers are not contiguous, as we show. We then give a sequential algorithm whose competitive ratio is also $O(\lg \sigma)$. The algorithm works also in the case of weighted items and non-contiguous item identifiers. Both algorithms are matched by a $\Omega(\lg \sigma)$-lower bound on the competitive ratio of any deterministic online algorithm, even sequential algorithms for unweighted contiguous SIC.

Several additional results are omitted for luck of space. One is a simple algorithm in 
the case of resource augmentation, that is 1-competitive when allowed to accept two items per conflict. Another is an oblivious $O(\lg \sigma / b)$-competitive algorithm for the generalized problem when $b$ items may survive each conflict. Finally, we present an alternative sequential algorithm whose competitiveness is expressed in terms of the depth of the interval structure, where depth is defined to be the maximal number of conflicts that any single item is involved in.

\subsection{Related Work}

The offline version of our problem, finding a maximum subset of points with no two in a common interval, is easily solvable in polynomial time (see Section 2). A related minimization problem is finding the minimum number of points intersecting all intervals, or alternatively minimum clique partition. A 2-competitive online algorithm for the latter problem is given and shown to be the best possible in [6].

Note the unusual characteristic of our problem is that the solution only decreases as more of the input arrives. Little is known about online maximization problems of this sort; the only related result we are aware of is [3].

A different dual problem is the interval selection problem, where we seek a maximum cardinality subset of disjoint intervals. In the online version, the intervals that arrive over time must be irrevocably accepted or rejected. Randomized algorithms for different cases are known $[9,1,2]$; the result closest in spirit to ours is an $O(\log m)$-competitive algorithm (originally for call control on the line) [1], where $m$ is the number of possible interval endpoints. In general, however, a $\Omega(n)$ lower bound holds for the competitive ratio of randomized algorithms [2], where $n$ is the number of intervals. Interval selection can be seen as an instance of scheduling with conflicts, which has been studied extensively (see, e.g., the surveys of $[7,10]$ ), but to the best of our knowledge, we are the first to consider online conflicts in the form of groups of consecutive items.

The problem of multi-packet frames (sketched above) was introduced in [8], where it is shown that if packet ordering is arbitrary (namely conflicts are not necessarily intervals), then the competitive ratio is $\Omega(\sigma)$ even for two-packet frames. A general framework that deals with transmission of multi-packet frames is described in [3]. The problem is modeled as an online version of Set Packing, nearly tight bounds of $\tilde{\Theta}(k \sqrt{\sigma})$ are proven on the competitive ratio of randomized algorithms for Online Set Packing, and a $\Omega\left(\sigma^{k-1}\right)$ deterministic lower bound is shown, where $k$ is the maximum size of a set and $\sigma$ is the maximum number of sets that contain the same element. In our terms, it is assumed there that each item is involved in up to $k$ conflicts, and conflicts need not be intervals.

\subsection{Paper Organization}

The remainder of this paper is organized as follows. In Section 2 we formalize the problem and present the basic arguments we use in analyzing our algorithms. We study oblivious algorithms in Section 3, and sequential algorithms are considered in Section 4. In Section 5 we prove a lower bound on the competitive ratio of online algorithms. Some concluding remarks are given in Section 6.

\section{Preliminaries and Basic Argument}

In this section we formalize the problem, define the concepts and notation we use, and present the basic argument we employ in the analysis of our algorithms. 


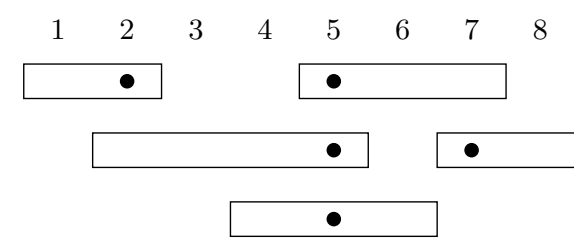

(a) $P=\{5\}$

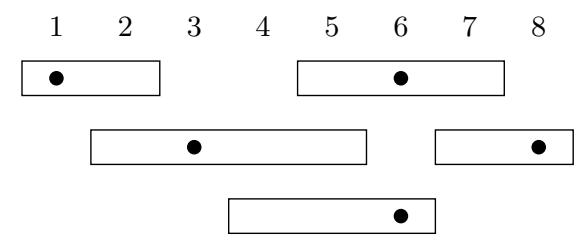

(b) $P=\{1,3,6,8\}$

Figure 1 An instance of SIC with two possible solutions. Rectangles represent conflicts, and dots represent items that were selected as survivors in conflicts. The instance contains eight items and five conflicts whose size is at most 4 (i.e., $\sigma=4$ ). In Figure 1a (top), only one item survives all conflicts, while an optimal solution can have four such items (Figure 1b).

\subsection{Problem Statement and Notation}

Scheduling with Interval Conflicts (abbreviated SIC) is defined as follows. There is a set $U$ of $n$ integer items. The input is a collection $\mathcal{C}$ of conflicts, where each conflict $C \in \mathcal{C}$ contains all items of $U$ within some interval on the real line. Namely, $C=U \cap[\min (C), \max (C)]$. In this paper we also consider the Contiguous Model, where $U$ is a set of consecutive integers. The size of the largest conflict is denoted by $\sigma_{\mathcal{C}}$, namely $\sigma_{\mathcal{C}} \stackrel{\text { def }}{=} \max \{|C|: C \in \mathcal{C}\}$ (the subscript is omitted when the instance is clear from the context). A feasible schedule is a set of items $P \subseteq U$ containing at most one item from any given conflict, i.e. $|P \cap C| \leq 1$ for every $C \in \mathcal{C}$. An item in $P$ is said to be a survivor of its conflicts, while the other items were eliminated. If item $i$ survives conflict $C \ni i$ by algorithm $A$, we say that $A$ delivers $i$ from $C$. The goal is to find a maximum cardinality feasible schedule, i.e. maximize the number of items surviving all their conflicts (see example in Figure 1).

In the weighted case, each item $i$ has a real-valued weight $w(i)>0$ and the objective is to find a maximum weight subset of weights satisfying the conflict constraints. For a set $S$ of items, $w(S) \stackrel{\text { def }}{=} \sum_{i \in S} w(i)$.

We consider two models of algorithms. In the oblivious model, the selection of a survivor from a conflict is a function of that conflict only, which allows for distributed conflict resolution. In the sequential model, conflicts arrive over time, i.e., they are ordered as a sequence $C_{1}, C_{2}, \ldots$, and the resolution of conflict $C_{t}$ may be a function of the full history $C_{1}, \ldots, C_{t}$.

We note that simple heuristics for SIC may perform poorly. For example, selecting the leftmost item in each given conflict is $\Omega(n)$-competitive as demonstrated by the instance in Figure 2. The same goes for the sequential strategy of picking the leftmost item among the items that were not eliminated in previous conflicts. (We assume that the top conflict is the first to arrive.)

\subsection{Characterizing Optimal Solutions}

The offline version of SIC can be reduced to maximum independent set in proper intervals graphs, which is solvable in polynomial time [5]. The reduction is as follows. First, remove all conflicts that are properly contained in other conflicts. It follows that there is a total order on the remaining conflicts, and therefore we may view each item as a node in a proper interval graph, where an interval is now a contiguous sequence of conflicts. Now finding an optimal schedule amounts to finding a maximum independent set in the above mentioned 


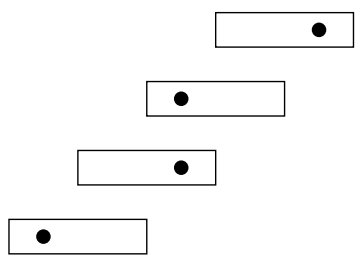

Figure 2 An instance of SIC with $n$ items and $n-1$ conflicts (all of size 2, i.e., $\sigma=2$ ). If one selects the leftmost item in each given conflict, only one item (number 1) survives all conflicts, while by always picking the odd-numbered items (represented by dots in the figure), one gets an optimal solution of size $\lceil n / 2\rceil$.

interval graph. Similarly, one may find a maximum independent set in an interval graph by solving an offline sIC instance.

We give a more direct description below. First, we provide an upper bound on the optimal solution due to duality. Let $\operatorname{OPT}(\mathcal{C})$ denote an optimal solution of SIC to instance $\mathcal{C}$, and let $U(\mathcal{C})=\bigcup_{C \in \mathcal{C}} C$ denote the set of items involved in conflicts in $\mathcal{C}$.

- Observation 1. For all $\mathcal{C}^{\prime} \subseteq \mathcal{C}$ : If $U\left(\mathcal{C}^{\prime}\right)=U(\mathcal{C})$, then $|\operatorname{OPT}(\mathcal{C}) \cap U(\mathcal{C})| \leq\left|\mathcal{C}^{\prime}\right|$.

Observation 1 motivates a simple polynomial offline algorithm for sIC. Briefly, the idea is to scan the item set from left to right (the examples in Figure 1 may help the reader), initially selecting the leftmost item. The next element selected, following a selected element $i_{j}$, is then inductively the leftmost element among those that are not in conflicts that contain $i_{j}$, i.e., $i_{j+1}=\min \left\{i^{\prime}: i^{\prime}>i_{j}\right.$ and $\left.\forall C,\left|\left\{i_{j}, i^{\prime}\right\} \cap C\right| \leq 1\right\}$. This forms a feasible solution, since for any consecutively chosen items $i_{j}$ and $i_{j+1}$, there is no conflict containing both $i_{j}$ and $i_{j+1}$. To prove that the selected elements constitute an optimal solution, let $C_{j}^{\prime}$ be the conflict that contains $i_{j}$ and $i_{j+1}-1$. Since $\bigcup_{j} C_{j}^{\prime}=U(\mathcal{C})$, optimality follows from Observation 1.

\section{Oblivious Algorithms}

In this section we consider oblivious algorithms. Oblivious algorithms are attractive because they can be implemented in a distributed system. The main result of this section is an oblivious algorithm for unweighted contiguous SIC, whose competitive ratio is $2 \lg \sigma$. We also show that if the instance is not contiguous, then no oblivious algorithm can be competitive.

\subsection{Oblivious Algorithm for Contiguous SIC}

In this section we present a simple $2 \lg \sigma$-competitive algorithm for unweighted contiguous sIC. We note that the algorithm needs not know $\sigma$ in advance.

The basic idea of the algorithm is to assign to each item a fixed priority, and the conflict resolution rule is to always prefer the item with the highest priority. Specifically, our algorithm, Priority, defines the priority of item $i$ by

$$
p(i) \stackrel{\text { def }}{=} \max \left\{\ell \in \mathbb{Z} \mid i \text { is divisible by } 2^{\ell}\right\} .
$$




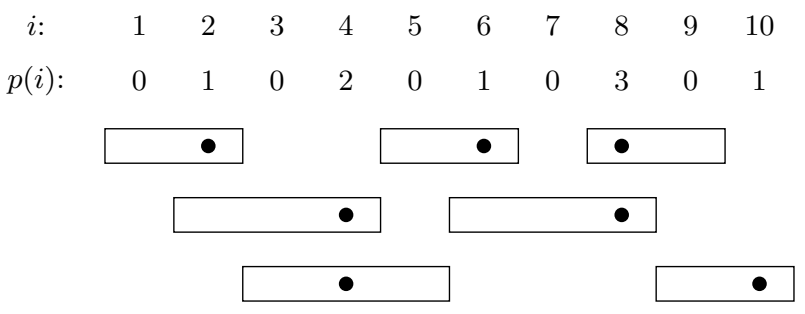

Figure 3 Execution of Algorithm Priority on an instance with ten items and seven conflicts. The dots represent the items that were chosen by the algorithm. The computed solution is $P=\{4,8,10\}$, while the optimum is $\{1,3,6,9\}$.

For example, if $i$ is odd, then $p(i)=0$, and if $i=2^{\ell}$ then $p(i)=\ell{ }^{1}$

One nice consequence of this definition is the following observation:

- Observation 2. If $i_{\ell}<i_{r}$ and $p\left(i_{\ell}\right)=p\left(i_{r}\right)=p$ for some $p$, then there exists $i_{\ell}<i<i_{r}$ such that $p(i)>p$.

Observation 2 implies that any conflict contains exactly one item with maximum priority, and hence Algorithm Priority is well-defined: Upon arrival of conflict $C$, the algorithm delivers the unique item with highest priority (as defined by (1)) among the items in $C$. See Figure 3 for an example. Note that the algorithm makes decisions without knowing or even estimating $\sigma$, and that it is completely distributed: the identity of the winner of a conflict is independent of other conflicts.

Next, observe that even though $\sigma$, the size of the largest conflict, is unknown, we need only to concern ourselves with $\lg \sigma$ priorities.

- Observation 3. Each conflict contains at most one item $i$ with $p(i) \geq \lg \sigma$.

Observation 3 implies that given conflicts whose length is bounded by $\sigma$, all priorities greater than or equal to $\lg \sigma$ are indistinguishable from the viewpoint of Algorithm Priority.

We now turn to prove that the competitive ratio of Algorithm Priority is at most $2 \lg \sigma$. We use the following concept.

- Definition 4. Let $\mathcal{C}$ be an instance and let $A$ be an algorithm for sic. A sequence of items $i_{0}, i_{1}, \ldots, i_{m}$ is an elimination chain of length $m$ if for all $0<j \leq m$ we have that item $i_{j}$ eliminates item $i_{j-1}$ when $A$ runs on $\mathcal{C}$.

Note that an elimination chain of length $m$ contains $m+1$ items, but implies the existence of $m$ conflict intervals. Elimination chains have the following property.

- Lemma 5. Let $\mathcal{C}$ be an instance and let $A$ be an algorithm for SIC. Suppose that $i_{0}, \ldots, i_{m}$ is an elimination chain for $\mathcal{C}$ under $A$. Then the interval $\left[i^{\prime}, i^{\prime \prime}\right]$ can be covered by $m$ conflicts, where $i^{\prime}=\min \left\{i_{j} \mid 0 \leq j \leq m\right\}$ and $i^{\prime \prime}=\max \left\{i_{j} \mid 0 \leq j \leq m\right\}$.

Proof. By Definition 4 , for any $0<j \leq m$ there exists a conflict $I_{j} \in \mathcal{I}$ such that $i_{j-1} \in I_{j}$ and $i_{j} \in I_{j}$. It follows, by induction on $m$, that $\bigcup_{j=1}^{m} I_{j} \supseteq\left[i^{\prime}, i^{\prime \prime}\right]$.

\footnotetext{
1 For an efficient implementation (in $\mathrm{AC} 0$ ), e.g. to use in routers, it suffices to extract the smallest bit set, using the bit-wise operations $(i$ XOR $(i-1))$ AND $i$.
} 
Lemma 5 implies the following consequence. Say that an algorithm is reasonable if it delivers an item from each conflict $C$, i.e. it does not eliminate items without a reason.

- Proposition 6. The competitive ratio of any reasonable algorithm for SIC is at most $2 m-1$, where $m$ is the length of the longest elimination chain of the algorithm.

Proof. Fix an instance $\mathcal{C}$. Say that an item $i^{\prime}$ is dominated by an item $i$ if there is an elimination chain that starts with $i^{\prime}$ and ends with $i$. For each item $i$, let $D(i)$ be the set of items dominated by $i$. Now, consider a reasonable algorithm $A$, and let $P$ be the set of items delivered by $A$ running on $\mathcal{C}$. Clearly, each item $i$ was either delivered by $A$, i.e., $i \in P$, or eliminated, in which case $i \in D\left(i^{\prime}\right)$ for some $i^{\prime} \in P$ (because $A$ is reasonable). In addition, we have by Lemma 5 that $D\left(i^{\prime}\right)$ can be covered by the conflicts of two elimination chains: one starting from $\min \left(D\left(i^{\prime}\right)\right)$ and one that starts from $\max \left(D\left(i^{\prime}\right)\right)$. Both of them end in the same interval. We can therefore conclude that the set of all items can be covered by $(2 m-1)|P|$ intervals. The result now follows from Observation 1.

Using Proposition 6 and Observation 3 we can easily bound the competitive ratio of Algorithm Priority.

- Theorem 7. The competitive ratio of Algorithm Priority is at most $2 \lg \sigma$.

Proof. By Observation 3 and the fact that under Priority an item $i$ is eliminated by an item $i^{\prime}$ if and only $p(i)<p\left(i^{\prime}\right)$, we have that the length of any elimination chain under Priority is at most $\lg \sigma$. The theorem therefore follows directly from Proposition 6 .

In the full version of the paper we explain how to extend Algorithm Priority to the capacitated case, where the number of survivors from a conflict may be some parameter $b \geq 1$.

\subsection{A Lower Bound for the Non-Contiguous Case}

One may wonder if a similar result holds in the non-contiguous case. This turns out to be far from the case. We argue that no deterministic oblivious algorithm is competitive in the general (non-contiguous) case even if $\sigma=2$.

- Theorem 8. The competitive ratio of any deterministic oblivious algorithm for SIC is $\Omega(n)$, even for the unweighted case and for $\sigma=2$.

Proof. Fix a deterministic oblivious algorithm ALG. By definition of obliviousness, the decision of ALG for a given conflict $C$ depends only on its items. Let $n$ be a number and let $N=2^{n}$. We 2-color the edges of an $N$-vertex clique $K_{N}$ as follows. Edge $\left(v_{i}, v_{j}\right)$, for $i<j$, is colored blue if ALG prefers $v_{i}$ over $v_{j}$, and otherwise red. By Ramsey's theorem [4], $K_{N}$ contains a monochromatic subgraph of $\log N=n$ vertices. If follows that there is either an increasing or a decreasing sequence of $n$ items $i_{1}, \ldots, i_{n}$ such that ALG prefers $i_{\ell}$ over $i_{\ell-1}$, for any $\ell \in\{2, \ldots, n\}$. We introduce the conflicts $\left\{i_{\ell-1}, i_{\ell}\right\}$, for $\ell \in\{2, \ldots, n\}$. Then, only $i_{n}$ will survive the execution of ALG, whereas $\left\{i_{\ell}: \ell\right.$ is odd $\}$ is a feasible solution of size $n / 2$.

\section{Sequential Algorithms}

In this section we present an $O(\lg \sigma)$-competitive sequential algorithm for sIC, extending algorithm Priority to the weighted and non-contiguous case. 
In describing our algorithm, we say that item $i$ fired item $i^{\prime}$ if $i$ was the first item to eliminate $i^{\prime}$. In the remainder of this section we say that an item $i_{0}$ is dominated by item $i_{m}$ if there is an elimination chain $i_{0}, i_{1}, \ldots, i_{m}$ such that $i_{j}$ fires item $i_{j-1}$, for every $j$. Let $D(i)$ be the set of items that are dominated by $i$. Note that if $i$ survives then $D(i) \neq \emptyset$, and in particular $i \in D(i)$. Observe that each item is dominated by exactly one surviving item, hence $D(i) \cap D\left(i^{\prime}\right)=\emptyset$ for each pair of surviving items $i$ and $i^{\prime}$.

We now describe the algorithm in the weighted case. Define the weight class of item $i$ to be $c(i)=\lfloor\lg w(i)\rfloor$, the base-2 logarithm of the item weight rounded down to an integer. Our algorithm is called Seq, and it proceeds as follows.

With each item $i$, we associate two values $\operatorname{left}(i)$ and $\operatorname{right}(i)$ (initially both zero), referred to as the left and right levels of $i$, respectively. When an interval $I$ arrives, the algorithm determines the highest weight class of active items in $I$. If there is only one active item of the highest weight class, it simply survives. Otherwise, let $l$ and $r$ be the leftmost and rightmost active items of the highest weight class. The algorithm compares left $(l)$, the left level of $l$, and $\operatorname{right}(r)$, the right level of $r$. If $\operatorname{left}(l)>\operatorname{right}(r)$, then $l$ survives and $\operatorname{right}(l)$ is set to $\operatorname{right}(r)+1$; otherwise, $r$ survives and $\operatorname{left}(r)$ is set to $\operatorname{left}(l)+1$. (The algorithm arbitrarily favors $r$, in case of a tie.) Notice that left $(i)$ and $\operatorname{right}(i)$ may increase and decrease during execution.

Fix some optimal solution OPT. The following upper bound is what motivates the numbering of the levels. Let $m_{i}$ be the larger of the levels of $i$, namely $m_{i}=\max \{\operatorname{left}(i), \operatorname{right}(i)\}$. Also, let $n_{i}=\max _{i^{\prime} \in D(i)} m_{i^{\prime}}$ be the largest level of an item in $D(i)$.

- Lemma 9. $w(\mathrm{OPT} \cap D(i))=O\left(n_{i} \cdot w(i)\right)$.

Proof. Let $l_{1}, \ldots, l_{t}$ be the sequence of items in $D(i)$ such that $l_{1}$ is the leftmost item in $D(i)$ and, inductively, $l_{j+1}$ is the item that fired item $l_{j}$. Observe that the sequence extends monotonically from left to $i$, with $l_{t}=i$. According to the survival rule of the algorithm, the weight classes of the items are monotonically non-decreasing, and for a pair of items $l_{j}$ and $l_{j+1}$ in the same weight class, the left levels are strictly increasing, namely $\operatorname{left}\left(l_{j+1}\right)>\operatorname{left}\left(l_{j}\right)$. It follows that there are at most $n_{i}$ items from the item set $\left\{l_{1}, \ldots, l_{t}\right\}$ in each weight class. Hence, the sum of the weights of the items $l_{1}, \ldots, l_{t}$ is bounded by

$$
\sum_{j=1}^{t} w\left(l_{j}\right)<2 \sum_{j=1}^{t} 2^{c\left(l_{j}\right)} \leq 2 n_{i} \sum_{c \leq c(i)} 2^{c}<2 n_{i} \cdot 2^{c(i)+1} \leq 4 n_{i} \cdot w(i) .
$$

Let $D^{-}(i)\left(D^{+}(i)\right)$ be the subset of items in $D(i)$ to the left (right) of $i$, up to and including $i$. That is, $D^{-}(i) \cup D^{+}(i)=D(i)$ and $D^{-}(i) \cap D^{+}(i)=\{i\}$. Partition $D^{-}(i)$ into ranges $\left[l_{j+1}, l_{j}\right]$, for $j=1, \ldots, t-1$. Observe that $l_{j+1}$ must be in the largest weight class among the items in the range $\left[l_{j}, l_{j+1}\right]$, for all $j=1, \ldots, t-1$. (Namely, if there was a item in $\left[l_{j}+1, l_{j+1}-1\right]$ belonging to a larger weight class, the largest such item could not have been eliminated without either $l_{j}$ or $l_{j+1}$ being also eliminated.) Since OPT can contain at most one item from each range $\left[l_{j}, l_{j+1}\right]$, it follows from $(2)$ that

$$
w\left(\mathrm{OPT} \cap D^{-}(i)\right)<2 \sum_{j=1}^{t} w\left(l_{j}\right) \leq 8 n_{i} \cdot w(i) .
$$

Applying the same arguments to $D^{+}(i)$ yields that $w($ OPT $\cap D(i)) \leq 16 n_{i} \cdot w(i)$, implying the lemma.

We now show that high levels imply very large intervals. 
- Lemma 10. $n_{i}=O(\lg \sigma)$.

Proof. Let $\hat{D}\left(i^{\prime}\right)$ be the set of items dominated by $i^{\prime}$ that are from the same weight class, $c\left(i^{\prime}\right)$, as $i^{\prime}$.

- Claim 1. $\left|\hat{D}\left(i^{\prime}\right)\right| \geq 2^{m_{i^{\prime}}}$, for any item $i^{\prime}$ in $D(i)$.

Proof. The proof is by induction on $m_{i^{\prime}}$. The base case $m_{i^{\prime}}=0$ is trivially true, since $i^{\prime} \in \hat{D}\left(i^{\prime}\right)$. For the inductive step, suppose that $m_{i^{\prime}} \geq 1$. Consider the most recent conflict $I$ that $i^{\prime}$ survived and in which an item from class $c\left(i^{\prime}\right)$ was fired. Let $l$ and $r$ be the leftmost and rightmost active items in $I$, respectively, such that $c(l)=c(r)=c\left(i^{\prime}\right)$, when $I$ was presented. Observe that $i^{\prime} \in\{l, r\}$. By the inductive hypothesis, $|\hat{D}(l)| \geq 2^{m_{l}}$ and $|\hat{D}(r)| \geq 2^{m_{r}}$. If $m_{i^{\prime}}=\max \left\{m_{l}, m_{r}\right\}$, then we are done. Suppose then that $m_{i^{\prime}}=\max \left\{m_{l}, m_{r}\right\}+1$, which happens only when $m_{l}=m_{r}$. Since $\hat{D}(l)$ and $\hat{D}(r)$ are disjoint, we have that

$$
\left|\hat{D}\left(i^{\prime}\right)\right| \geq|\hat{D}(l)|+|\hat{D}(r)| \geq 2^{m_{l}}+2^{m_{r}}=2^{m_{i^{\prime}}} .
$$

and the claim follows.

- Claim 2. $\hat{D}\left(i^{\prime}\right)$ is covered by at most $2 m_{i^{\prime}}$ intervals.

Proof. Let $l_{1}, l_{2}, \ldots, l_{t}$ be the sequence of items defined such that $l_{1}$ is the leftmost item in $\hat{D}\left(i^{\prime}\right)$ and, inductively, $l_{j+1}$ is the item that fired $l_{j}$, for $j=1, \ldots, t-1$. Also, let $I_{j}$ be the interval presented upon which $l_{j+1}$ fired $l_{j}$, for $j=1, \ldots, t-1$. Clearly, $I_{1}, \ldots, I_{t-1}$ cover the items to the left of $l_{t}=i^{\prime}$, up to and including $i^{\prime}$. According to the survival rule of the algorithm, the left levels of the items are strictly increasing. It follows that $t \leq m_{i^{\prime}}+1$. By symmetry, $m_{i^{\prime}}$ intervals also cover the items in $\hat{D}\left(i^{\prime}\right)$ to the right of $i^{\prime}$.

We resume with the proof of Lemma 10. Let $i^{\prime} \in D(i)$ such that $n_{i}=m_{i^{\prime}}$. By the two claims above, some interval covers at least $\left|\hat{D}\left(i^{\prime}\right)\right| /\left(2 m_{i^{\prime}}\right) \geq 2^{m_{i^{\prime}}-1} / m_{i^{\prime}}$ items. Hence, $\sigma \geq 2^{m_{i^{\prime}}-1} / m_{i^{\prime}}$, or $n_{i}=m_{i^{\prime}} \leq \lg \sigma(1+o(1))$.

The following theorem is now immediate from Lemmas 9 and 10 when observing that the sets $\{D(i): i$ survived $\}$ partition the set $U$ of items.

- Theorem 11. The competitive ratio of the oblivious algorithm Seq for the weighted and non-contiguous case is $O(\lg \sigma)$.

\section{A Lower Bound on the Competitive Ratio}

In this section we show that the competitive ratio of any deterministic online algorithm for contiguous SIC is $\Omega(\lg \sigma)$. Our lower bound construction is sequential, namely the conflicts arrive one by one, and the algorithm knows the complete history when a new conflict arrives. Since any algorithm for oblivious SIC can be used in the sequential model, the lower bound holds for oblivious SIC as well.

Fix a deterministic online algorithm $A$. Based on the way $A$ picks items to survive conflicts, we construct in an online fashion a sequence of conflicts along with an optimal scheduling denoted by OPT. To facilitate the description, define a conflict $I$ to be active with respect to algorithm $A$ if upon arrival, $I$ contains a item that was not already eliminated by $A$ in previous conflicts. W.l.o.g., we consider only algorithms that always deliver an item from an active interval. 


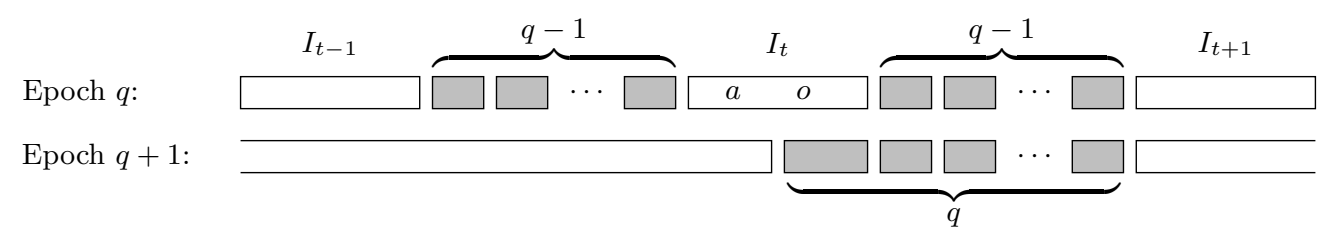

Figure 4 Construction of epoch $q+1$ : The case where $a<o$. Gray boxes represent positive intervals. $I_{t}$ is split into two parts: the left part is combined with $I_{t-1}$, while the right part becomes a positive interval.

In general, there can be conflicts that are active with respect to $A$, OPT, or both. We call a conflict interval neutral if it is active with respect to both $A$ and OPT, and positive if it is active with respect to OPT only (there will be no "negative" intervals in our construction).

The conflict sequence consists of a sequence of epochs satisfying the following epoch invariant:

- In each epoch, all conflicts are disjoint and their union is $\{1, \ldots, n\}$.

- The set of items delivered by $A$ and by OPT from epoch $q \geq 1$ are disjoint.

- In epoch $q$ there are $q-1$ positive intervals between any two consecutive neutral intervals. Note that the last property means that after epoch $q$, the optimal number of surviving items is $q$ times larger than the number of items delivered by $A$.

We now describe the construction of epochs inductively. Assume that $n$ is an even integer. The first epoch consists of $n / 2$ intervals of size 2 : for every $t \in[1, n / 2]$, the $t$ th interval is $[2 t-1,2 t]$. Let $A_{1}$ be the set of items that are delivered by the algorithm after the first epoch. Clearly $\left|A_{1}\right|=n / 2$. The optimal solution is the complement of $A_{1}$, namely $\mathrm{OPT}_{1}=\{1, \ldots, n\} \backslash A_{1}$. It is straightforward to verify that the epoch invariant holds for $q=1$ (the last property follows from the fact that all intervals in epoch 1 are neutral).

The more interesting part is the inductive step. Let $A_{q}$ and $\mathrm{OPT}_{q}$ be the set of active items with respect to $A$ and OPT, respectively, immediately after epoch $q$. Assume that the invariant holds for epoch $q$. We construct epoch $q+1$ and $\mathrm{OPT}_{q+1}$ as follows. Number the neutral intervals of epoch $q$ sequentially $I_{1}, I_{2}, \ldots$, starting from the leftmost neutral interval. This numbering skips the positive intervals between neutral intervals. Let $I_{t}=\left[\ell_{t}, r_{t}\right]$ be the $t$ th neutral interval, where $t$ is even. We break $I_{t}$ into two parts as follows. Let $a$ and $o$ be the indices of items that are delivered from $I_{t}$ by $A_{q}$ and $\mathrm{OPT}_{q}$, respectively in epoch $q$. We proceed by two cases. If $a<o$, then we introduce the conflict interval $\left[o, r_{t}\right]$ and extend $I_{t-1}$ to the right up to $o-1$ (see Figure 4). Otherwise, if $a>o$, then we introduce the conflict interval $\left[\ell_{t}, o\right]$ and extend $I_{t+1}$ to the left up to $o+1$ (see Figure 5). Notice that an odd neutral interval from epoch $q$ can be either extended to the left, or to the right, or in both directions, or not extended at all. Finally, positive intervals from epoch $q$ that are not covered by the above intervals are added to epoch $q+1$. Figure 6 illustrates a complete example.

It remains to determine $\mathrm{OPT}_{q+1}$. Let $I_{t}^{\prime}$ be the extended version of an odd interval $I_{t}$ from epoch $q$. $\mathrm{OPT}_{q+1}$ will deliver the active item from $I_{t}$. Since $I_{t}$ does not intersect any even neutral interval, $\mathrm{OPT}_{q+1}$ may deliver an item in any part of an even neutral interval that was added to epoch $q+1$. Also, since $I_{t}$ does not intersect any positive interval from epoch $q, \mathrm{OPT}_{q+1}$ may deliver an item in any positive interval that was not merged with an odd neutral interval from epoch $q$.

The first and second properties of the invariant are clearly satisfied by the construction. 


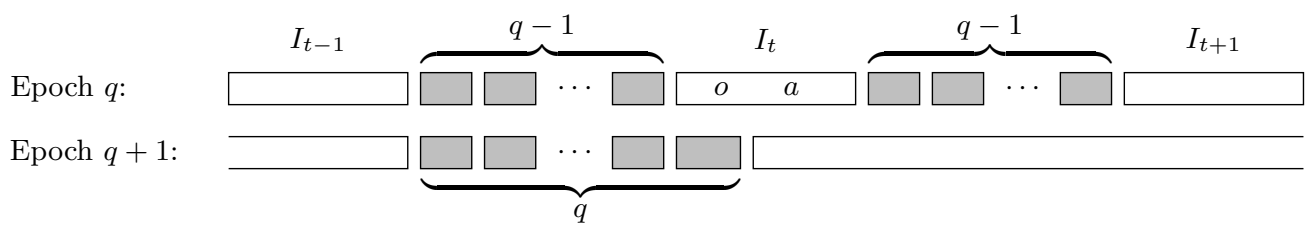

Figure 5 Construction of epoch $q+1$ : The case where $a>o$. Gray boxes represent positive intervals. $I_{t}$ is split into two parts: the right part is combined with $I_{t+1}$, while the left part becomes a positive interval.

\begin{tabular}{|c|c|c|c|c|c|c|c|c|c|c|c|c|c|c|c|c|c|}
\hline 1 & 2 & 3 & 4 & 5 & 6 & 7 & 8 & 9 & 10 & 11 & 12 & 13 & 14 & 15 & 16 & 17 & 18 \\
\hline$a$ & $O$ & $O$ & $a$ & $a$ & $O$ & $O$ & $a$ & $a$ & $O$ & $a$ & $O$ & $O$ & $a$ & $O$ & $a$ & $a$ & $O$ \\
\hline$a$ & $O$ & $O$ & & $a$ & $o$ & $O$ & & $a$ & $O$ & & $O$ & $O$ & $a$ & $O$ & & $a$ & $O$ \\
\hline$a$ & $O$ & & & & $o$ & $O$ & & $a$ & $O$ & & $O$ & $O$ & & & & $a$ & $O$ \\
\hline
\end{tabular}

Figure 6 The lower bound construction with $n=18$ and three epochs. The gray boxes represent positive intervals.

To see that the last property of the invariant holds, observe that any extended odd neutral interval remains neutral. We claim that there are $q$ positive intervals between any consecutive neutral intervals. Let $I_{t}$ be an even neutral interval that was split in the construction of epoch $q+1$. If $a<o$ the interval $\left[o, r_{t}\right]$ is positive because $\left[o, r_{t}\right] \cap A_{q}=\emptyset$. Moreover, all positive intervals between $I_{t}$ and $I_{t+1}$ remain as they were. Similarly, if $a>o$ the interval $\left[\ell_{t}, o\right]$ is positive, because $\left[\ell_{t}, o\right] \cap A_{q}=\emptyset$, and all positive intervals between $I_{t}$ and $I_{t-1}$ are left unchanged. Hence, there are $q$ positive intervals between any two consecutive neutral intervals in epoch $q+1$.

The following lemma bounds the size of intervals.

- Lemma 12. Let $\sigma_{q}$ be the maximum interval size in epoch $q$. Then $\sigma_{q} \leq 2 \cdot 5^{q-1}$.

Proof. By induction on the number of epochs. In the base case (epoch 1 ), $\sigma_{1}=2$. For the inductive step, observe that an interval in the epoch $q+1$ may consist of (i) an odd neutral interval, (ii) parts of two even neutral intervals, (iii) $2(q-1)$ positive intervals. Since positive intervals that are created in epoch $q^{\prime}$ are of size smaller than $\sigma_{q^{\prime}-1}$ and due to the inductive hypothesis, we have that

$$
\sigma_{q+1}<\sigma_{q}+2 \sigma_{q}+2 \sum_{q^{\prime}=1}^{q-1} \sigma_{q^{\prime}} \leq 3 \sigma_{q}+2 \sum_{q^{\prime}=1}^{q-1} \sigma_{q^{\prime}}<5 \sigma_{q},
$$

and the lemma follows.

We can now prove the lower bound.

- Theorem 13. The competitive ratio of any deterministic online algorithm for sequential SIC is $\Omega(\lg \sigma)$, even in the contiguous case.

Proof. Let $A$ be a deterministic algorithm. Construct instance $\mathcal{I}$ as described above. The epoch invariant implies that after $q$ epochs, $|\mathrm{OPT}(\mathcal{I})| \geq q(|A(\mathcal{I})|-1)$. Hence, the competitive ratio of $A$ is $\Omega(q)$. Let $\sigma$ be a given parameter. By Lemma 12 we have that $\sigma_{q} \leq 2 \cdot 5^{q-1}$, and therefore, setting $q=\left\lfloor\log _{5}(\sigma / 2)\right\rfloor=\Omega(\lg \sigma)$ we have $\sigma_{q} \leq \sigma$, and the result follows. 


\section{Conclusion}

In this paper we have introduced the problem of scheduling with interval conflicts and proved tight bounds on the competitive ratio of online algorithms to solve them. It would be interesting to consider other conflict topologies, and to understand to which degree randomness can help.

\section{References}

1 Baruch Awerbuch, Yair Bartal, Amos Fiat, and Adi Rosén. Competitive non-preemptive call control. In Proc. 5th SODA, pages 312-320, 1994.

2 Unnar Thor Bachmann, Magnús M. Halldórsson, and Hadas Shachnai. Online selection of intervals and $t$-intervals. In Proc. 11th SWAT, volume 6139 of Lecture Notes in Computer Science, pages 383-394, 2010.

3 Yuval Emek, Magnús M. Halldórsson, Yishay Mansour, Boaz Patt-Shamir, Jaikumar Radhakrishnan, and Dror Rawitz. Online set packing and competitive scheduling of multi-part tasks. In Proc. 29th PODC, pages 440-449, 2010.

4 Paul Erdős and G. Szekeres. A combinatorial problem in geometry. Compositio Math., 2:463-470, 1935.

5 M.C. Golumbic. Algorithmic graph theory and perfect graphs. Academic Press, New York, 1980.

6 Jerzy W. Jaromczyk, Andrzej Pezarski, and Maciej Slusarek. An optimal competitive algorithm for the minimal clique covering in circular arc graphs. In Proc. 19th EWCG, 2003.

7 David Karger, Cliff Stein, and Joel Wein. Scheduling algorithms. In Mikhail J. Atallah, editor, Algorithms and Theory of Computation Handbook. CRC Press, 1998.

8 Alexander Kesselman, Boaz Patt-Shamir, and Gabriel Scalosub. Competitive buffer management with packet dependencies. In Proc. 23rd IPDPS, pages 1-12, 2009.

9 R.J. Lipton and A. Tomkins. Online interval scheduling. In Proc. 5th SODA, pages 302-311, 1994.

10 Jiri Sgall. On-line scheduling. In Online Algorithms, volume 1442 of Lecture Notes in Computer Science, pages 196-231, 1996. 